\title{
液相剥离法制备石墨烯及在导热硅橡胶中的应用
}

\author{
史智慧 ${ }^{1,2}$ ，李 莹 ${ }^{1}$ ，间璐 ${ }^{1}$ ，曹暳真 ${ }^{1}$ \\ (1. 中国科学院 上海硅酸盐研究所, 特种无机涂层重点实验室, 上海 200050; 2. 中国科学院大学, 北京 100049)
}

摘 要: 采用高速剪切机液相剥离法, 在胆酸钠的水溶液中将鳞片石墨剥离, 离心得到石墨烯分散液。AFM、TEM、 Raman 表征结果发现, 剥离出的石墨烯厚度小于 4 层, 尺寸大约在 $2 \sim 3 \mu \mathrm{m}$, 高质量缺陷少 $\left(I_{\mathrm{D}} / I_{\mathrm{G}} \approx 0.15\right)$ 。将石墨烯 分散液冷冻干燥后与银粉共同添加到硅橡胶中, 制备出导热硅橡胶。利用稳态热流法测试导热硅橡胶的导热系数发 现，当添加 $3 \mathrm{vol} \%$ 石墨烯时，复合材料的导热系数由未添加石墨烯时的 $4.900 \mathrm{~W} /(\mathrm{m} \cdot \mathrm{K})$ 提高到 $12.367 \mathrm{~W} /(\mathrm{m} \cdot \mathrm{K})$ 。综上 所述，通过液相剥离法成功制备出缺陷较少的少层石墨烯，能够与银粉协同提高导热硅橡胶的导热系数。

关 键 词: 液相剥离法; 石墨烯; 热导率

中图分类号: TQ174 文献标识码: A

\section{Graphene: Prepared by High Speed Shearing-assisted Exfoliation and Application in Thermal Conductive Silicone}

\author{
SHI Zhi-Hui ${ }^{1,2}$, LI Ying $^{1}$, YAN Lu ${ }^{1}$, CAO Yun-Zhen ${ }^{1}$
}

\begin{abstract}
(1. Key Laboratory of Inorganic Coating Materials CAS, Shanghai Institute of Ceramics, Chinese Academy of Sciences,
\end{abstract} Shanghai 200050, China; 2. University of Chinese Academy of Sciences, Beijing 100049, China )

\begin{abstract}
Graphene dispersion was produced by centrifugation after exfoliating the graphite in aqueous solutions containing sodium cholate as a surfactant with high speed shearing machine. The exfoliated graphene (thickness $\leqslant 4$ layers) showing large lateral size of around $2-3 \mu \mathrm{m}$ and high quality $\left(I_{\mathrm{D}} / I_{\mathrm{G}} \approx 0.15\right)$ was characterized by atomic force microscopy (AFM), transmission electron microscopy (TEM), and Raman spectroscopy. After freeze drying, Graphene was added in the silver-silicone rubber to produce a hybrid graphene-metal thermal conductive silicone rubber, and steady-state heat flow method was applied to study the thermal conductivity of the composite. It was found that as filler loading fraction of graphene increased from 0 to $3 \mathrm{vol} \%$, thermal conductivity of siliver-silicone rubber dramatically improved from $4.9 \mathrm{~W} /(\mathrm{m} \cdot \mathrm{K})$ to $12.367 \mathrm{~W} /(\mathrm{m} \cdot \mathrm{K})$. This result suggests that little-defect graphene with few layers prepared by exfoliating can significantly enhance the thermal conductivity of silicone rubber by synergy effect when combined with silver powder.
\end{abstract}

Key words: liquid exfoliation ; graphene ; thermal conductivity

导热硅橡胶在电子元件散热领域应用广泛，它可 以填充在电子元件与散热器之间, 使电子元件产生的 热量更快速地传递到散热器，而硅橡胶基体中由于没 有自由电子或较为有序的晶体结构, 使其不能有效地 进行热传导, 导热系数一般只有 $0.12 \sim 0.20 \mathrm{~W} /(\mathrm{m} \cdot \mathrm{K})$, 因此一般在有机硅基础胶中加入高导热填料来提高
其导热率 ${ }^{[1]}$ 。Zhou 等 ${ }^{[2]}$ 通过填充不同粒径的 $\mathrm{Al}_{2} \mathrm{O}_{3}$, 使硅橡胶的热导率提高到 $1.45 \mathrm{~W} /(\mathrm{m} \cdot \mathrm{K})$; $\mathrm{Ji}$ 等 ${ }^{[3]}$ 填充 $55 \mathrm{vol} \%$ 低温等离子改性后的 $\mathrm{BN}$, 使硅橡胶导热系数 达到 $2.40 \mathrm{~W} /(\mathrm{m} \cdot \mathrm{K})$ 。但是随着芯片的集成度不断提 高和功耗密度不断增大, 传统的导热填料如 $\mathrm{Al}_{2} \mathrm{O}_{3}$ 、 BN 已不能满足现在市场对热管理材料的要求。并且 
电子产品微型化、轻型化以及高效化发展使散热问 题更加严峻，需开发导热性能更加优异的散热材料 来解决散热问题 ${ }^{[4-6]}$ 。

石墨烯具有良好的电学与光学性能, 单层厚度 仅为 $0.335 \mathrm{~nm}$, 热导率为 $5000 \mathrm{~W} /(\mathrm{m} \cdot \mathrm{K})$, 室温下电 子迁移率高达 $2 \times 10^{5} \mathrm{~cm}^{2} /(\mathrm{V} \cdot \mathrm{s})^{[7-9]}$ 。将其作为纳米增 强组分加入聚合物中, 可以有效改善聚合物的机 械、电、热等性能, 已成为聚合物基纳米复合材料 研究的热点。但是, 目前应用于复合材料填料的石 墨烯 ${ }^{[10-16]}$, 大多数是采用氧化还原法制备。该方法 虽然能够大规模工业化生产石墨烯，但是制备的石 墨烯结构破坏较严重, 使石墨烯的各种优异性能大 打折扣。而机械剥离法, 虽然理论上可以制备无缺 陷高品质石墨烯, 但产量却非常低。化学气相淀积 法能够制备大面积石墨烯, 但是薄膜的分离和转移 工艺难度很大, 且多用于微电子产业。因此发展一 种可以大量制备高性能石墨烯的新方法尤为重要。

Coleman 等 ${ }^{[17]}$ 发现, 在特定溶剂中, 通过超声波剥 离可直接制备高品质石墨烯, 是一种有望规模化生 产高质量石墨烯的方法。

银粉的导热系数为 $427 \mathrm{~W} /(\mathrm{m} \cdot \mathrm{K})$, 是一种很好 的非绝缘的导热填料。Cui 等 ${ }^{[18]}$ 向环氧树脂中添加 $85 \mathrm{wt} \%$ 球状银粉, 导热系数增加到 $6 \sim 7 \mathrm{~W} /(\mathrm{m} \cdot \mathrm{K})$ 。但 是银粉由于还原性较强, 在加温固化过程中表面容 易生成一层氧化银, 而氧化银的电阻是银粉的 $6 \times$ $10^{6}$ 倍, 这就大大增加了界面热阻, 从而影响银粉作 为高导热填料的导热效率。YANG 等 ${ }^{[19]}$ 对银粉进行 碘处理，发现银粉表面形成了许多不稳定存在的 $\mathrm{Ag} / \mathrm{AgI}$ 纳米团簇, 固化前后电阻降低了 $60 \%$, 并且 $\mathrm{Ag} / \mathrm{AgI}$ 纳米团簇中的纳米银颗粒比大块银粉具有 更强的还原性, 在固化过程中优先被氧化, 阻止了 银粉表面的氧化, 因此增强了银粉表面活性, 并显 著降低了界面热阻。因此, 将经过碘处理的银粉作 为主要导热填料, 并利用石墨烯作为增强组分, 有 希望制备出性能优异的导热复合材料。

本工作首先通过液相剥离法制备高质量的少层 石墨烯, 并对其结构、形貌进行表征; 然后将石墨 烯与经过碘处理的银粉作为填料制备导热硅橡胶复 合材料, 并探究不同体积分数的石墨烯对复合材料 热导率的影响, 为制备高导热系数的导热硅橡胶提 供实验与理论研究基础。

\section{1 实验方法}

\section{1 实验原料}

天然鳞片石墨, 300 目, 南京先丰纳米有限公司
生产; 水合胆酸钠(SC), Aladdin 公司生产; 乙烯基 聚二甲基硅氧烷、氢基聚二甲基硅氧烷、铂催化剂, Sigma-Aldrich 公司生产; 片状银粉(SF-107), 昆明 诺曼电子材料公司生产; 碘、乙醇, 上海国药化学试 剂有限公司生产。

\section{2 液相剥离石墨烯}

首先在烧杯中加入 $600 \mathrm{~mL}$ 去离子水, $9.00 \mathrm{~g}$ 鳞 片石墨, $0.06 \mathrm{~g}$ 胆酸钠; 然后将混合液在冰水浴中以 剪切机 $6000 \mathrm{r} / \mathrm{min}$ 的转速高速剪切 $6 \mathrm{~h}$, 每隔 $30 \mathrm{~min}$ 移动一下烧杯位置, 得到石墨烯的分散液; 最后取 分散液以 $8000 \mathrm{r} / \mathrm{min}$ 的速度在 SIGMA 3-30K 高速离 心机离心分离 $30 \mathrm{~min}$, 取上层清液, 冷冻干燥成石 墨烯粉末。

液相剥离石墨烯所使用的高速剪切设备为 Silverson model L5M, 图 1(a c c) 为高速剪切机及其 转头的照片，定子与转子之间有 $200 \mu \mathrm{m}$ 的间隙, 在高速剪切过程中, 鳞片石墨在其中不断被减薄, 高速离心最终得到如图 1(d)所示的石墨烯分散液。

\section{3 石墨烯/银粉硅橡胶复合材料的制备}

称取一定量的银粉，在乙醇中超声 $2 \mathrm{~h}$ 后过滤， 然后称取碘粒(银粉与碘的质量比为 $100: 0.2$ ), 并 溶于乙醇中, 将碘液逐滴加入银粉中, 机械搅拌 $1 \mathrm{~h}$ 后, $50^{\circ} \mathrm{C}$ 烘干，密封保存在阴暗处。将烘干的经过碘处 理后的银粉与石墨烯粉末用研针研磨均匀, 取 $5.24 \mathrm{~g}$ 硅橡胶 A 组分, 用移液管滴入 $20 \times 10^{-6}$ 的铂 催化剂, 真空行星摚拌混合 $20 \mathrm{~min}$, 加入 $4.76 \mathrm{~g}$ 硅 橡胶 B 组分, 真空摚拌 $20 \mathrm{~min}$, 将石墨烯、银粉混 合后的填料分批加入硅橡胶中, 真空摚拌。将物料 转移到模具中, $3 \mathrm{kN}$ 真空干压 $1 \mathrm{~h}$ 后置于烘箱中 $150^{\circ} \mathrm{C}$ 固化 $1 \mathrm{~h}$ 。

\section{4 石墨烯/银粉硅橡胶复合材料的表征}

利用激光共焦拉曼光谱仪 (Horiba Lab-Ram iHR550, 波长为 $632.8 \mathrm{~nm}$, 测量波数范围 $1000 \sim 3000 \mathrm{~cm}^{-1}$, 步幅 $10 \mathrm{~cm}^{-1} / \mathrm{s}$ 。) 对样品进行拉曼 光谱测试。利用扫描电子显微镜 (HITACHI S-3400N)、透射电子显微镜(JEOL JEM-2100)对样 品的形貌和结构进行观察。利用 BRUKERDimension Icon 原子力显微镜对石墨烯的厚度和二维尺 寸进行表征, 测量模式为轻敲模式, 扫描范围为 $20 \mu \mathrm{m} \times 20 \mu \mathrm{m}$, 分析软件为 Nanoscope Analysis 1.5。利用导热系数测试仪(台湾瑞领 LW9389 导 热仪, 测试标准 ASTM D5470)测试样品的导热 系数, 每种样品测试 3 个不同的厚度拟合出导热 系数。 


\section{2 结果与讨论}

\section{1 石墨烯的表征}

采用 TEM 对石墨烯在溶剂中分散形态和结构 进行分析, 在测试过程中, 将稀释一定倍数的石 墨烯分散液沉积在铜网上, 并在自然环境中干 燥。图 1(e)为液相剥离并经冷冻干燥得到的石墨烯 粉末的 TEM 照片, 图 1(f)是对应红色区域的电子衍 射图案, 标记的衍射区域祄度较弱, 可判断该处的 石墨烯层数较少, 但确切的层数却难以分辨, 颜色 较深区域的石墨烯有一定程度的折叠, 对选区进行 电子衍射分析表明, 清晰地显示出石墨烯的六角形 衍射几何, 且没有向环扩展的趋势, 可知衍射区域 为制备良好的石墨烯晶体, 最内层衍射强度较次内 层大很多, 这是单层石墨烯的特征。

为了进一步分析制备的少层石墨烯的剥离程度, 采用原子力显微镜轻敲模式进行研究。在制备样品 过程中, 先将石墨烯分散液稀释至近乎透明, 再将 其沉积在新鲜云母片上, 室温下自然干燥后进行测 试。图 $1(\mathrm{~g}, \mathrm{~h})$ 是液相剥离得到的石墨烯 AFM 图片 及其高度轮廓, 从图中可以看出, 在 $10 \mu \mathrm{m} \times 10 \mu \mathrm{m}$ 的扫描范围内, 分布了几十片石墨烯, 平均尺寸约 为 $2 \mu \mathrm{m}$, 大小比较均匀, 图中白色小点为分散液烘 干后残留的胆酸钠晶体。图 1(h)为三片石墨烯的厚 度图, 可以看出厚度约为 $0.6 \mathrm{~nm}$, 这是由于在自然 环境下挥发溶剂, 可能会导致石墨烯片与基底之 间吸收少部分水或残留少量的溶剂分子, 使测试 的石墨烯厚度有所增加, 这与文献中报道的胆酸 钠溶液剥离得到的单层石墨烯厚度一致 ${ }^{[16]}$ 。为了进 一步分析液相剥离法制备剥离产物的真实组成与结 构, 在石墨烯 AFM 图片中对 60 片石墨烯片进行统 计整理, 图 $1(i, j)$ 为石墨烯片平均厚度和大小的分布 直方图, 由图可知, 石墨烯片的厚度在 $0.6 \sim 3.0 \mathrm{~nm}$ 之间, 约 1 4 层, 尺寸在 $0.5 \sim 3.0 \mu \mathrm{m}$ 之间。结果表 明, 实验通过液相剥离法成功制备了少层甚至单 层石墨烯。

由图 2 中可见, 石墨烯样品分别在 1355、1590 和 $2700 \mathrm{~cm}^{-1}$ 处出现了 $\mathrm{D}$ 峰、 $\mathrm{G}$ 峰和 $2 \mathrm{D}$ 峰。 $\mathrm{D}$ 峰为 布里渊区的边界模, 它的出现可能是由于经过剧烈 的剪切过程后石墨烯片层内的碳原子杂化状态发生 了变化, 即石墨烯片层内部分碳原子由 $\mathrm{sp}^{2}$ 杂化状 态转变为 $\mathrm{sp}^{3}$ 杂化状态导致 $\mathrm{sp}^{2}$ 区域减少而造成的。 $\mathrm{G}$ 峰产生于 $\mathrm{sp}^{2}$ 碳原子的面内振动, 是与布里渊区中 心双重简并的 iTO 和 iLO 光学声子相互作用产生的,
具有 $E_{2 g}$ 对称性, 是单层石墨烯中唯一的一个一阶 拉曼散射过程。2D 峰是与 $\mathrm{K}$ 点附近的 iTO 光学声 子发生两次谷间非弹性散射产生的, 拉曼位移约为 $\mathrm{D}$ 峰的 2 倍, 但它的产生与缺陷无关, 并非 D 峰的 倍频信号。据文献报道, 单层石墨烯的 2D 峰强度大 于 $\mathrm{G}$ 峰, 并具有完美的单洛伦兹峰形, 随着层数的 增加 $2 \mathrm{D}$ 峰的半峰宽增大并向高波数位移(蓝移), 而 图中得到的 Raman 峰形与文献中报道的 3 4 层石墨 烯一致, 这可能是干燥过程中石墨烯有一定团聚造成 的 ${ }^{[20]}$ 。 $\mathrm{D}$ 峰与 $\mathrm{G}$ 峰的强度比是表征石墨烯缺陷密度的 重要参数, $I_{\mathrm{D}} / I_{\mathrm{G}} \approx 0.15$, 表明通过高剪切过程剥离得到 的石墨烯的结构保存较完整, 含有很少的缺陷。

\section{2 碘处理前后银粉的 SEM 及 EDS 表征}

图 3 为碘处理前后片状银粉的 SEM 照片, 从图 中可以发现, 碘处理前片状银粉表面光滑, 表面无 明显颗粒存在，片状银粉按照质量比 $\left(\mathrm{Ag}: \mathrm{I}_{2}=500: 1\right)$ 处理后，在其表面形成了纳米团簇。对这些纳米团 簇进行 EDS 分析, 发现纳米团簇内 $\mathrm{Ag}$ 与 I 的比值 不同，由于 $\mathrm{AgI}$ 不稳定，见光易分解为纳米银颗粒， 从而形成 $\mathrm{Ag} / \mathrm{AgI}$ 纳米团簇, 它比大块银粉具有更 强的还原性, 能够在加热固化过程中优先与氧气反 应, 阻止银粉表面的氧化, 进而降低界面热阻, 改 善银粉表面活性。

\section{3 石墨烯添加量与硅橡胶复合材料导热系 数的关系}

目前国内测试导热硅胶大多根据美国 ASTM D5470 标准测定，该标准适用于厚度在 0.02 10.00 mm 间的均相材料的热阻测试。研究表明, 试样热阻与 热流流过的距离(即试样的厚度)成比例, 测试一系 列不同厚度试样的热阻, 然后以热阻对试样厚度作 图, 拟合直线的斜率倒数可得到试样的热导率。本 工作测试了同一配方三种不同厚度试样的热阻, 进 而得到试样的热导率。

将经过碘处理的银粉作为填料, 制备出一系 列不同体积分数的导热硅橡胶, 并测试复合材料 的热导率。从图 4 (a)中可以发现, 当银粉的填充比 例从 $25 \mathrm{vol} \%$ 增加到 $35 \mathrm{vol} \%$ 时, 硅橡胶的导热系数 由 $1.20 \mathrm{~W} /(\mathrm{m} \cdot \mathrm{K})$ 增大到 $4.99 \mathrm{~W} /(\mathrm{m} \cdot \mathrm{K})$ 。刚开始, 导 热系数增长比较缓慢, 但当银粉填充比例达到 $30 \mathrm{vol} \%$ 后，导热系数增长幅度变大，这表明银粉填 充比例在 30vol\%时, 导热网络开始逐渐形成, 因此 导热系数增长幅度变大。继续添加银粉, 胶体粘度急 剧增加, 不能够完全固化, 因此银粉最优的填充比 例定为 $35 \mathrm{vol} \%$ 。 

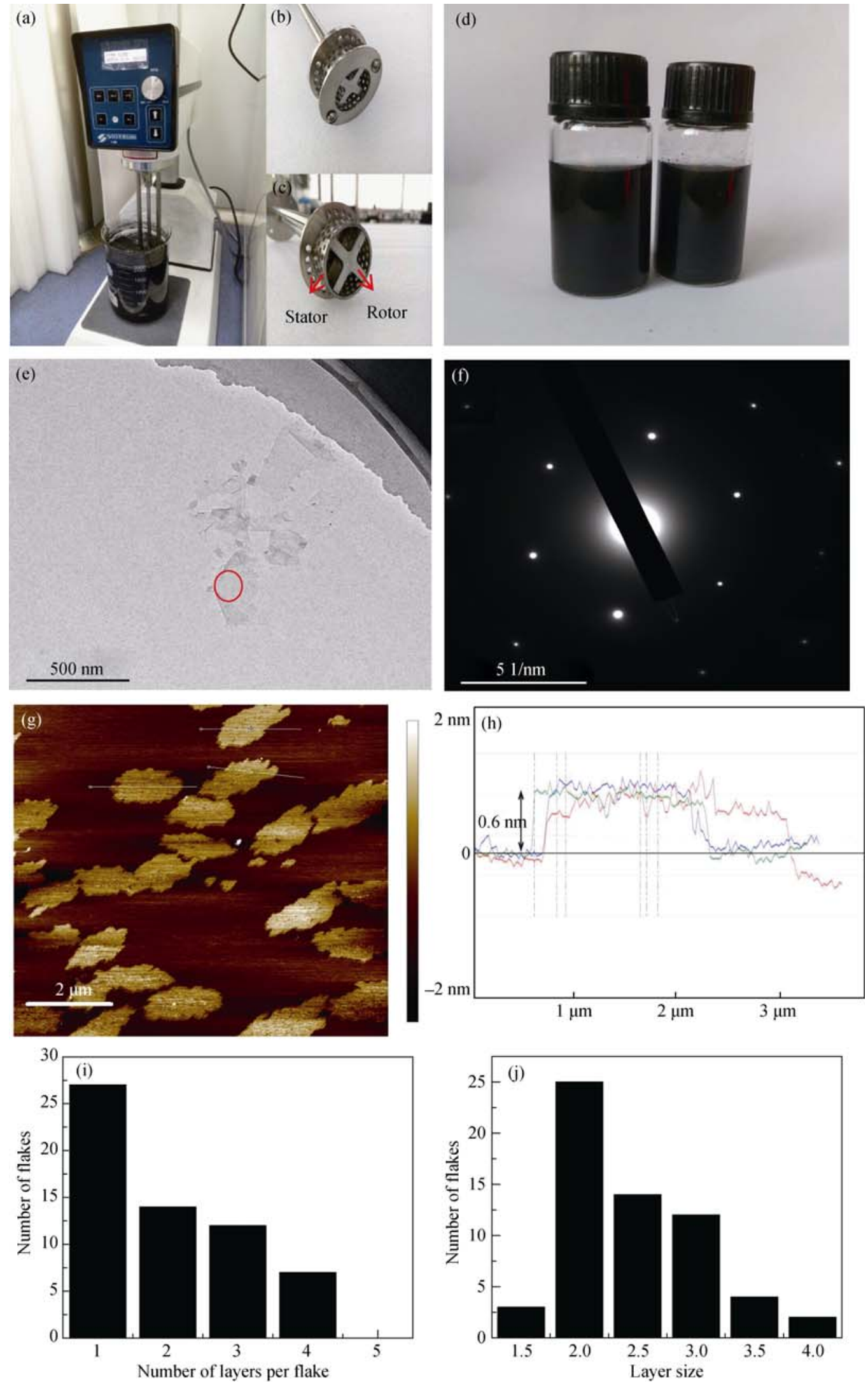

图 1 (a c) 高速剪切机及其转头实物图, (d)离心得到的石墨烯分散液照片, ( $\sim \mathrm{f})$ 石墨烯的 TEM 照片及局部电子衍射图; $(\mathrm{g} \sim \mathrm{h})$ 液 相剥离得到的石墨烯 AFM 及其高度轮廓, (i j ) 石墨烯片厚度和横向尺寸的分布直方图

Fig. 1 (a-c) A Silverson model L5M high-shear mixer with mixing head in 21 beaker of graphene dispersion; (d) Graphene dispersion produced by shear exfoliation; (e-f) TEM image of individual nanosheets and its electron diffraction pattern; (g-h) AFM height profiles of graphene dispersion $(10 \mu \mathrm{m} \times 10 \mu \mathrm{m})$; (i-j) Histogram of number of layers and lateral size of graphene flakes 


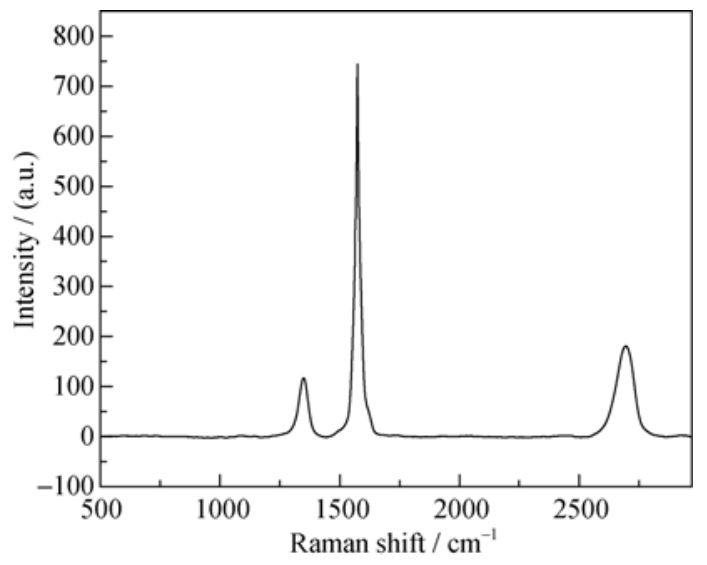

图 2 冷冻干燥后的石墨烯的拉曼光谱图

Fig. 2 Raman spectrum of graphene after freeze drying

将上述通过液相剥离法得到的少于 4 层、尺寸在 2 3 $\mu \mathrm{m}$ 的石墨烯粉末添加到银粉硅橡胶中。从图 4 (b) 中可以发现，当石墨烯添加量分别为 $0 、 0.4 \mathrm{vol} \%$ 、 $1 \mathrm{vol} \% 、 2 \mathrm{vol} \% 、 3 \mathrm{vol} \%$ 时，复合材料的热导率分别 为 $4.991 、 8.299 、 10.192 、 11.73 、 12.367 \mathrm{~W} /(\mathrm{m} \cdot \mathrm{K})$ 。 相对于银粉硅橡胶复合材料的热导率分别提高了 1.66 倍、 2.04 倍、 2.35 倍、 2.48 倍。

单一碘处理银粉作为导热填料, 导热通路在体 积分数为 $35 \mathrm{vol} \%$ 时逐渐形成, 从图 4(b)可知, 石墨
烯的添加显著地提高了复合材料的热导率。添加 $0.5 \mathrm{vol} \%$ 石墨烯时，硅橡胶导热率增长幅度较大，但 添加 $1 \mathrm{vol} \% 、 2 \mathrm{vol} \%$ 时, 硅橡胶的导热率增长幅度变 小, 而添加 $3 \mathrm{vol} \%$ 后, 导热率增长又逐渐趋向于缓 慢。这可能是由于单独添加 $35 \mathrm{vol} \%$ 碘处理银粉时, 石墨烯大的比表面积及本身的褶皱结构，使其与基 体的接触面积增大，进而降低了界面热阻，加入少 量的石墨烯有助于桥接银粉, 彼此形成有效的导热 通路, 因此导热率增长幅度较大。而随着体系中导 热通路的形成, 继续添加石墨烯效果不太明显, 导 热率增长幅度相对缓慢。

Wang 等 ${ }^{[21]}$ 采用非平衡分子动力学模拟方法研 究了石墨烯/聚乙烯纳米复合材料的界面导热性能, 主要考察了石墨烯层数、尺寸对界面热阻的影响, 研究结果表明: 随着石墨烯层数的增加, 界面热导 下降, 界面热导随着石墨烯尺寸的增大而逐渐增 大。在单层石墨烯中声子的传输模式为弹道声子模 式，声子之间碰撞较少，但对于多层石墨烯，声子 会与层间的粒子发生碰撞散射，同时多层石墨烯之 间存在声子的倒逆散射，所以导致石墨烯的热导率 下降, 并且随着石墨烯层数的增加, 声子散射越多, 热导率下降就越多。随着石墨烯尺寸的增大，石墨
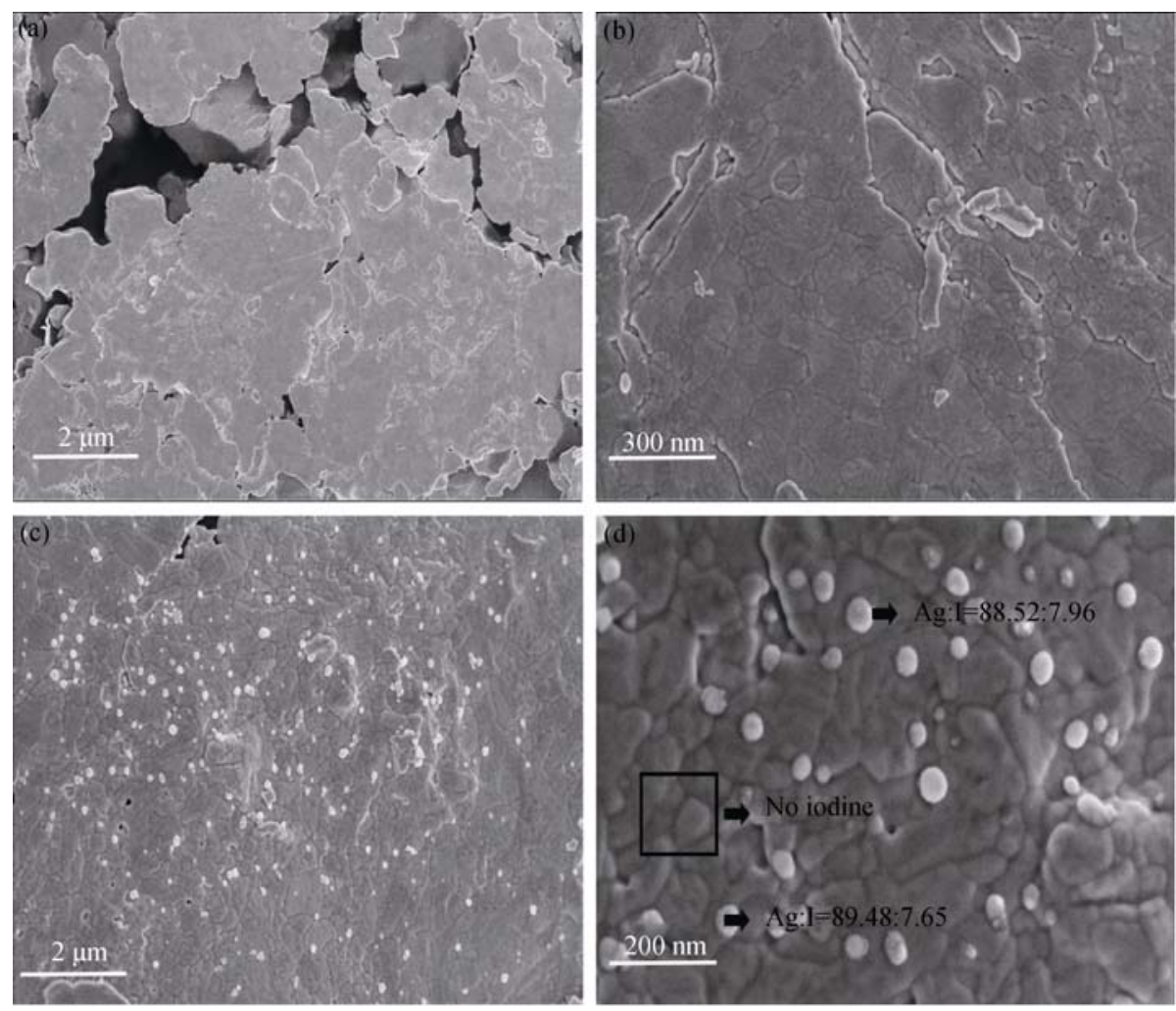

图 3 碘处理前 $(a \sim b)$ 和后(c $\sim d)$ 片状银粉的 SEM 照片

Fig. 3 SEM images of the silver microflake samples before (a-b) and after (c-d) iodine modification 

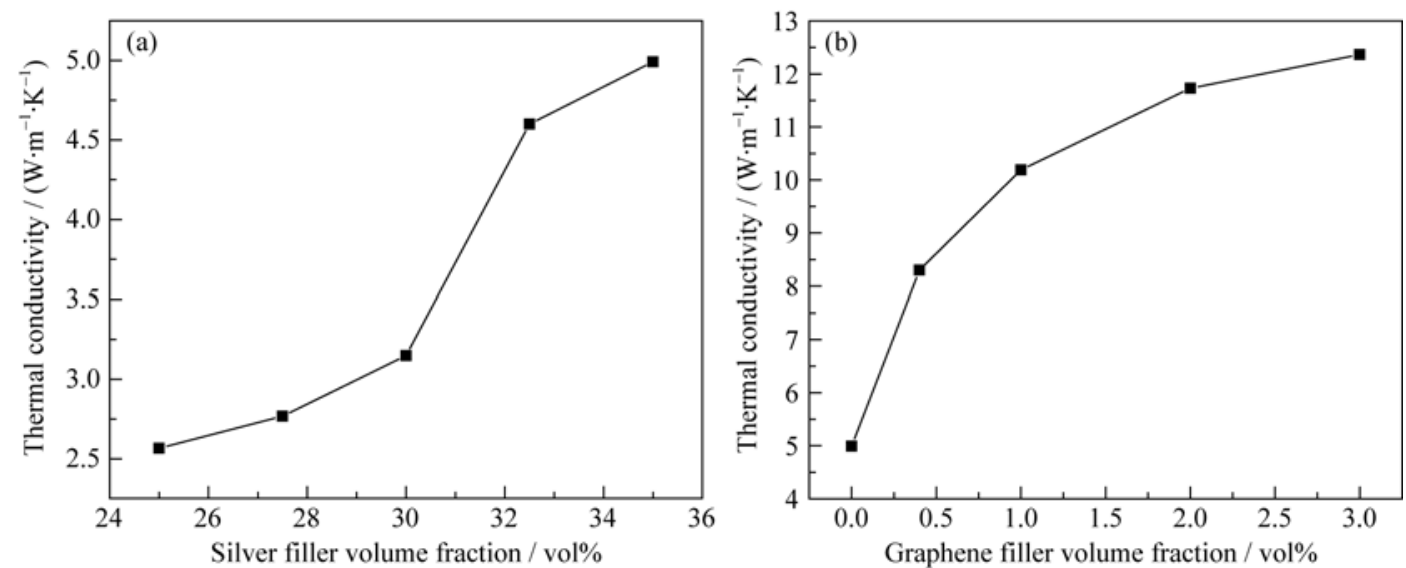

图 4 银粉添加量与硅橡胶复合材料(a)和石墨烯添加量与银粉硅橡胶复合材料(b)导热系数关系图

Fig. 4 Thermal conductivity of the samples vs various silver filler loadings (a) and the samples vs various graphene filler loadings (b)

烯中长波长声子逐渐被引发, 在更低的振动频率范 围内产生了新的振动模式，并与高分子原子的振动 模式产生进一步的叠加，使得界面热导逐渐增大。

\section{3 结论}

通过高速剪切作用，在胆酸钠的水溶液中从 鳞片石墨成功剥离出结构较为完整、尺寸均匀的 单层(或数层)石墨烯, 冷冻干燥后添加到银粉硅 橡胶中，与碘处理后的银粉协同增强了复合材料 的导热系数。当添加体积分数为 $3 \mathrm{vol} \%$ 的石墨烯 时，复合材料的导热系数增大了 2.48 倍，导热系 数达到 $12.367 \mathrm{~W} /(\mathrm{m} \cdot \mathrm{K})$, 说明液相剥离法制备得 到的石墨烯具有良好的导热性能，在导热复合材 料中有很好的应用前景。

\section{参考文献:}

[1] SHIT SUBHAS C, SHAH PATHIK. A review on silicone rubber. National Academy Science Letters, 2013, 36(4): 355-365.

[2] ZHOU WEN-YING, QI SHU-HUA, TU CHUN CHAO. Effect of the particle size of $\mathrm{Al}_{2} \mathrm{O}_{3}$ on the properties of filled heat-conductive silicone rubber. Journal of Applied Polymer Science, 2007, 104(2): $1312-1318$.

[3] JI TUO, ZHANG LI-QUN, WANG WEN-CAI, et al. Cold plasma modification of boron nitride fillers and its effect on the thermal conductivity of silicone rubber/boron nitride composites. Polym. Compos., 2012, 33(9): 1473-1481.

[4] KONG S M, MARIATTI M, BUSFIELD J. Effects of types of fillers and filler loading on the properties of silicone rubber composites. Journal of Reinforced Plastics and Composites, 2011, 30(13): 1087-1096.

[5] CHUNG D.Thermal interface materials. Journal of Materials Engineering and Performance, 2001, 10(1): 56-59.

[6] SIM L C, RAMANAN S, ISMAIL H, et al. Thermal characterization of $\mathrm{Al}_{2} \mathrm{O}_{3}$ and $\mathrm{ZnO}$ reinforced silicone rubber as thermal pads for heat dissipation purposes. Thermochimica Acta, 2005, 430(1): 155-165.

[7] CASTRO NETO, GUINEA F, PERES N M R, et al. The elec tronic properties of graphene. Reviews of Modern Physics, 2009, 81(1): 109-162.

[8] BALANDIN ALEXANDER A, GHOSH SUCHISMITA, BAO WEN-ZHONG, et al. Superior thermal conductivity of single-layer graphene. Nano Letters, 2008, 8(3): 902-907.

[9] NOVOSELOV K S, GEIM A K, MOROZOV S V, et al. Electric field effect in atomically thin carbon films. Science, 2004, 306(5696): 666-669.

[10] KROTO H W, HEATH J R, O'BRIENS C, et al. C-60- buckminsterfullerene. Nature, 1985, 318(6042): 162-163.

[11] EIZENBERG M, BLAKELY J M. Carbon monolayer phase condensation on Ni(111). Surface Science, 1979, 82(1): 228-236.

[12] BERGER C, SONG Z, LI T, et al. Ultrathin epitaxial graphite:2D electron gas properties and a route toward graphene-based nanoelectronics. The Journal of Physical Chemistry B, 2004, 108(52): 19912-19916.

[13] BERGER CLAIRE, SONG ZHI-MIN, LI XUE-BIN, et al. Electronic confinement and coherence in patterned epitaxial graphene. Science, 2006, 312(5777): 1191-1196.

[14] STANKOVICH S, PINER R D, CHEN X, et al. Stable aqueous dispersions of graphitic nanoplatelets via the reduction of exfoliated graphite oxide in the presence ofpoly(sodium4-styrenesulfonate). Journal of Materials Chemistry, 2006, 16(2): 155-158.

[15] STANKOVICH SASHA, DIKIN DMITRIY A, PINER RICHARD D, et al. Synthesis of graphene-based nanosheets via chemical reduction of exfoliated graphite oxide.Carbon, 2007, 45(7): 1558-1565.

[16] HERNANDEZ Y, NICOLOSI V, LOTYA M, et al. High-yield production of graphene byliquid-phase exfoliation of graphite. Nature Nanotechnology, 2008, 3(9): 563-568.

[17] PATON KEITH R, VARRLA E, BACKES C, et al. Scalable production of large quantities of defect-free few-layer graphene by shear exfoliation in liquids. Nature Materials, 2014, 13(6): 624-630.

[18] CUI H W, FAN Q, LI D S, et al. Formulation and characterization of electrically conductive adhesives for electronic packaging. Journal of Adhesion, 2013, 89(1): 19-36.

[19] YANG CHENG, XIE YU-TAO, YUEN MATTHEW MING-FAI, et al. silver surface iodination for enhancing the conductivity of conductive composites. Advanced Functional Materials, 2010, 20(16): $2580-2587$.

[20] LOTYA MUSTAFA, KING PAUL J, KHAN UMAR, et al. High-concentration, surfactant-stabilized graphene dispersions. ACS Nano, 2010, 4(6): 3155-3162.

[21] WANG JIAN, MIAO RUI-ZHEN, ZHAO GUI-ZHE, et al. Interface heat conductance performance of graphene/PE:a molecular dynamics simulation. Journal of Molecular Science, 2016, 32(2): 156-161. 